\title{
LA PLANIFICACIÓN FAMILIAR COMO HERRAMIENTA BÁSICA PARA EL DESARROLLO
}

\author{
FAMILY PLANNING AS A BASIC TOOL FOR DEVELOPMENT
}

Miguel Gutiérrez $z^{1,2, a}$

Comisión de Alto Nivel de Salud Sexual y Reproductiva, Colegio Médico del Perú. Lima, Perú.

Pathfinder International Oficina de Perú. Lima, Perú.

Médico ginecólogo obstetra; magíster en género, sexualidad y salud reproductiva.

Recibido: 03-07-13; Aprobado: 21-08-13

\begin{abstract}
RESUMEN
El concepto de la planificación familiar (PF), tradicionalmente, ha estado y está muy relacionado con la salud sexual y reproductiva. En la Conferencia Internacional de Población y Desarrollo llevada a cabo en El Cairo en 1994, la PF es reconocida como una estrategia de salud, que bien aplicada puede contribuir al desarrollo de las naciones. A través de los últimos años se ha podido constatar que los beneficios no solo están dirigidos a prevenir embarazos no deseados y, con ello, prevenir abortos inducidos y la muerte materna, sino también mejora la salud infantil, mejora el acceso a la educación de los niños en el mundo; favorece el empoderamiento de la mujeres, no solo en el campo laboral sino también en otras áreas como en lo político y, con ello, el concepto de género; contribuye a disminuir la pobreza estimulando el desarrollo económico y el nivel de vida de las personas. Es decir, que contribuye a lograr no solamente uno sino los ocho objetivos de desarrollo del milenio para el año 2015. A pesar de la evidencia de sus beneficios, aún persisten barreras sociales, culturales, políticas o religiosas que hacen que exista una necesidad insatisfecha en planificación familiar, que no se respete el derecho de las personas a elegir y decidir el número de hijos que desean tener, y que impiden el disfrute de una sexualidad sana.
\end{abstract}

Palabras clave: Planificación familiar; Salud sexual y reproductiva; Embarazo en adolescencia (fuente: DeCS BIREME).

\begin{abstract}
The family planning (FP) concept has traditionally been, and is closely related to, sexual and reproductive health. At the International Conference on Population and Development held in Cairo in 1994, FP is recognized as a health strategy that, properly applied, can contribute to the development of nations. Over the past years we have found that the benefits are not only aimed at preventing unwanted pregnancies, there by preventing induced abortions and maternal death, but it also improves child health, facilitating access to education for children in the world, it promotes the empowerment of women not only in the workplace but also in other areas such as politics and thus, the concept of gender; it helps to reduce poverty by stimulating economic development and increasing the standard of living of people. This means that it not only contributes to one but to the eight Millennium Development Goals by 2015. Despite the evidence of its benefits, there are still social, cultural, political or religious barriers that cause an unmet need in Family Planning, which does not respect the right of individuals to choose and decide the number of children they want, and prevent the experience of a healthy sexuality.
\end{abstract}

Key words: Family Planning; Sexual and reproductive health; Pregnancy in adolescence (source: MeSH NLM).

\section{INTRODUCCIÓN}

La PF es un término que nos induce a pensar en una estrategia muy simple o sencilla que puede ayudar a las familias a tener conductas reproductivas adecuadas para que puedan conseguir sus objetivos de desarrollo saludable en la mejor forma. En parte, es verdad, pero realmente la PF es una estrategia que va mucho más allá de la familia, es un concepto que esta íntimamente ligado al desarrollo de las naciones.

Los temas reproductivos, en la actualidad y desde tiempos muy remotos, no solo han preocupado a las familias sino que empezó por ser una preocupación del propio individuo como persona, quien se dio cuenta que el ejercicio de su sexualidad, en determinadas circunstancias, generaba un embarazo en las mujeres y el nacimiento de un nuevo individuo. La preocupación individual, en los últimos tiempos, se ha transformado en una preocupación mundial, cuando el número de personas en el mundo es cada vez mayor, y la reducción de recursos naturales se relaciona con efectos sobre las mejores condiciones de habitabilidad de todas las personas en el mundo ${ }^{(1,2)}$.

Es en este contexto que se desarrolla el concepto moderno de la PF como una propuesta que debe influir

Citar como: Gutiérrez M. La planificación familiar como herramienta básica para el desarrollo. Rev Peru Med Exp Salud Publica. 2013;30(3):465-70 . 
no solo en mejorar la salud sexual y reproductiva de las personas, sino también la educación, en aliviar la pobreza, mejorar el ambiente, y en el desarrollo económico de las naciones; es decir, en un concepto amplio que debe ser tomado en cuenta por quienes tienen la responsabilidad de dirigir nuestros gobiernos, y que son quienes tienen que ver a la PF desde la perspectiva de considerarla como una herramienta básica para lograr el desarrollo de las naciones de una manera sostenible.

Consideraciones como las del neomaltusianismo han hecho que los Estados se preocupen por tener políticas de población. En el siglo pasado el ingreso del concepto de control de la natalidad en la década de 1970, fuertemente ligado al concepto demográfico $\mathrm{y}$, posteriormente, modificado por el concepto de PF, menos agresivo pero cuyo significado era muy similar, y que prácticamente se mantuvo hasta la Conferencia Internacional de Población y Desarrollo (CIPD) Ilevada a cabo en la ciudad de El Cairo, Egipto en septiembre del año $1994{ }^{(3)}$, donde se reformula el concepto de PF, y se liga al desarrollo, a la equidad de género y al respeto de los derechos humanos, específicamente al de los derechos sexuales y reproductivos.

En esta conferencia, la PF fue reconocida como una estrategia de salud, importante y fundamental, que aplicada en forma adecuada puede contribuir al desarrollo de los pueblos en el mundo. El énfasis se centró en la persona y, como tal, se reconocieron sus derechos a la salud y su salud sexual y reproductiva. Actualmente es considerada como un elemento promotor de los derechos humanos.

Por lo tanto, si el derecho a la salud es un derecho de todo individuo, es deber de los Estados garantizar que todos puedan tener, desde su nacimiento, las mejores condiciones que permitan el logro de personas saludables. Tener acceso a los alimentos, al vestido, al agua segura, a los servicios de salud de más alta calidad con programas de promoción y prevención, con una amplia variedad de medicamentos, y métodos anticonceptivos disponibles y accesibles. Es decir, tener acceso a una PF es también un derecho de todos y es deber del Estado garantizarla.

\section{PLANIFICACIÓN FAMILIAR COMO HERRAMIENTA BÁSICA PARA EL DESARROLLO}

La PF, en este nuevo concepto, si bien permite a las familias que puedan contar con metodologías que contribuyan a limitar su tamaño, para lograr una mejor distribución de sus recursos, no deja de lado a la persona como individuo, que tiene todo el derecho de hacer uso de los métodos anticonceptivos más efectivos para tener una mejor salud sexual, un disfrute sexual sin riesgos $y$, por ende, una mejor salud reproductiva. Por lo tanto, se involucra directamente con todos los elementos que forman parte de una población: la persona, la familia, las comunidades y los países, y eso le permite insertarlo en un concepto amplio de salud sexual y salud reproductiva incorporado en el nuevo paradigma de población y desarrollo, dejando de lado las metas exclusivamente demográficas.

La Constitución Política del Perú (4), aprobada por referéndum el año 1993 (un año antes de la CIPD en El Cairo), menciona en el Capítulo II artículo 6, que la política nacional de población tiene como objetivo difundir y promover la paternidad y maternidad responsables. Reconoce como un derecho fundamental el derecho de las familias y de las personas a decidir; sin embargo, no se vincula los beneficios de tener un programa adecuado de PF con el desarrollo de la nación. Según lo manifestado por el secretario general de las Naciones Unidas el año 2010, actualmente la PF es considerada como una de las mejores inversiones que los gobiernos y donantes pueden realizar en materia de desarrollo humano y económico, incluida la reducción de la pobreza, el crecimiento económico, la salud y el desarrollo infantil, el empoderamiento de las mujeres y los retos medioambientales.

Por otro lado, al prevenir los embarazos se promueve la igualdad de hombres y mujeres, tanto en el área profesional como laboral. Además, el prevenir la deserción escolar permite a las mujeres terminar su educación, tener una mejor capacitación y, con ello, acrecentar sus salarios, y así mejorar la situación de sus familias. Logrando empoderar a las mujeres.

\section{SALUD}

Todos los beneficios que podemos tener con un adecuado programa de PF se pierden cuando no se tienen cubiertas las necesidades de PF; es decir, en el aspecto sanitario, al no poder prevenir un embarazo que no es planificado, de acuerdo con las cifras del estudio de Allan Gutmacher en el año 1994, el 60\% ${ }^{(5,6)}$ se convertirá en un embarazo no deseado cuya posibilidad mayor es la de acabar en un aborto, que por las consideraciones legales que existe en Latinoamérica y, específicamente en el Perú, donde solamente el aborto terapéutico no está penalizado, la mayor parte de estos abortos serían de tipo inseguro, con grave riesgo para la salud de las mujeres, incluso la muerte ${ }^{(7)}$.

De hecho, la última comunicación sobre la mortalidad materna en el Perú llevado a cabo en el período del 
2002 al 2011 por la Dirección General de Epidemiología del Ministerio de Salud, nos dice que el aborto es la tercera causa de muerte materna directa con el 10,9\% de los casos ${ }^{(8)}$. Debemos también tener en cuenta que muchas mujeres que no fallecen por causa de un aborto inducido, quedan con enfermedades crónicas infecciosas, menopausia precoz por extirpación de ovarios, dolores crónicos, etc.

Además, no debemos olvidar que la PF, mediante los métodos anticonceptivos de barrera, ayuda a la prevención de infecciones de transmisión de sexual, incluidos el papiloma virus (PVH), el VIH y muchas otras infecciones cuya propagación anual en millones de personas y sus consecuencias en la salud son consideradas como un problema de salud pública.

También la PF contribuye a mejorar la salud infantil ya que permite que las mujeres, con un espaciamiento óptimo de los embarazos, dediquen más tiempo a los recién nacidos, con una mejor alimentación y un mejor desarrollo, preparados para una mejor educación.

\section{EDUCACIÓN}

Si no se cubre las necesidades de PF se puede tener un elevado costo social que incluye el aumento de la deserción escolar a causa de un embarazo no planificado. En las niñas que abandonan la escuela se observa una menor productividad del trabajo y, por ende, menor crecimiento de su economía; así como una estimación de menor ingreso futuro en el mercado del trabajo en comparación con las que tienen un nivel de escolaridad completo. En Latinoamérica, si bien los factores económicos para el abandono escolar son importantes, también se mencionan frecuentemente el embarazo y la maternidad ${ }^{(9)}$.

Está comprobado que el uso de métodos anticonceptivos entre mujeres y niñas aumenta el acceso a la educación, como ya hemos visto, muchas adolescentes con un embarazo se ven obligadas a abandonar la escuela. En los países del África Subsahariana, entre un 8 a $25 \%$ abandonan el colegio por causa de un embarazo no planificado. Por otro lado, las chicas que van a la escuela utilizan más los métodos anticonceptivos ${ }^{(10)}$

\section{GÉNERO Y EMPODERAMIENTO DE LAS MUJERES}

Poder planificar el número de hijos y cada cuánto tiempo los tendrán, da mayor capacidad de ahorro, de trabajo y de aumentar sus ingresos y planificar sus vidas, pudiendo invertir no solo en educación sino también en su formación profesional, una mayor preparación para el campo laboral, y para cubrir otras necesidades ${ }^{(3)}$.

\section{POBREZA Y DESARROLLO ECONÓMICO}

Con relación a la erradicación de la pobreza y el crecimiento económico podemos decir que la PF estimula el desarrollo económico y la mejora del nivel de vida ${ }^{(11,12)}$. Los países con menores tasas de fecundidad y de crecimiento de la población han experimentado mayor productividad, más ahorro y más inversión. El "bono demográfico" es la etapa de la transición demográfica en la que la población infantil se ha reducido y hay un grupo mayor de personas en edad de trabajar, entre 15 a 59 años, como efecto de la reducción de la tasa de fecundidad, las cuales tendrán que mantener a un grupo de mayor edad que no tiene capacidad de trabajar ${ }^{(13)}$. Los grupos asiáticos han visto crecer sus economías y disminuir sus niveles de pobreza a partir de la reducción de sus familias. Entre el 30 al 50\% del crecimiento económico de Asia entre el año 1965 al 1990 se ha atribuido a la dinámica demográfica, fuertemente influenciada por la planificación familiar ${ }^{(14)}$.

Algunos sostienen que existe una correlación entre la tasa de uso de métodos anticonceptivos y el PBI per cápita de los países (OMS 2010). Otros como Barot sostienen que si bien la reducción de la fecundidad por sí sola no impulsa el desarrollo económico sí es necesaria, y sostienen que un crecimiento entre el 25 al $40 \%$ del crecimiento económico en los países es atribuible a menores tasas de fecundidad. Por otro lado, disminuye la presión sobre los servicios públicos y los recursos sanitarios, incluyendo la vivienda, el empleo, y el agua potable ${ }^{(10)}$.

En conclusión, con relación a los Objetivos del Milenio, la PF interviene en todos los objetivos que se han propuesto alcanzar ${ }^{(15)}$. Es decir:

OD1: contribuye con mitigar la pobreza y acelera el desarrollo socioeconómico;

OD2: contribuye a garantizar que todos los niños vayan a la escuela;

OD3: promueve la equidad de género;

OD4: puede reducir la mortalidad infantil;

OD5: disminuye la mortalidad materna;

OD6: puede desacelerar la propagación del VIH/SIDA; OD7: puede ayudar a proteger el medioambiente.

\section{NECESIDADES INSATISFECHAS DE PLANIFICACIÓN FAMILIAR}

En los países en vías de desarrollo una de cada cuatro mujeres se encuentra entre los 15 a 49 años; es decir, en edad reproductiva. De ellas, 867 millones no desearían quedar embarazadas, espaciar los 
nacimientos o limitar sus embarazos futuros. Unas 645 millones están utilizando métodos modernos de anticoncepción y se calcula que unas 222 millones no usan ningún método moderno de anticoncepción, o utilizan métodos tradicionales y se encuentran con una necesidad insatisfecha de PF (16).

Las razones de no utilizar métodos anticonceptivos, a pesar de no desear tener un embarazo, pueden ser sociales, culturales o políticas. Muchas veces motivadas por los grupos religiosos o porque los hacedores de política no entienden la real importancia de la PF o no están de acuerdo y, por lo tanto, no garantizan el acceso a la anticoncepción moderna a todos los grupos etarios; por ejemplo, la restricción a grupos de adolescentes, o la falta de disponibilidad de anticonceptivos, no por hacer una compra inapropiada sino por carecer de políticas de distribución y supervisión permanente.

Un ejemplo claro, que se recoge en una reciente publicación, señala que desde que Alejandro Toledo asumió la presidencia de la república el Ministerio de Salud fue dominado por elementos conservadores religiosos, que se reflejó en un bloqueo al acceso a los métodos anticonceptivos, dicha posición fue asumida como respuesta a las políticas del gobierno de Fujimori (17).

Por otro lado, no debemos dejar de reconocer que hay factores individuales que también influyen en esta necesidad insatisfecha, como por ejemplo la sensación de tener poco riesgo de un embarazo, como ocurre en los adolescentes; o no estar informados adecuadamente y no entender los mensajes claves para la prevención de un embarazo, como ocurre después de un aborto, que muchas mujeres creen que es difícil quedar nuevamente embarazada, o cuando por mala información continúan teniendo relaciones sexuales no protegidas después de haber tomado la anticoncepción de emergencia, o tener relaciones sexuales durante la amenorrea posparto creyéndose protegidos porque "aún no le viene la regla".

En un estudio llevado a cabo el año 2006 con mujeres hospitalizadas en el Instituto Materno Perinatal, que tenían embarazos no deseados, se determinó que la principal razón para no usar métodos anticonceptivos era el temor por los efectos secundarios (92\%), siendo la subestimación del riesgo del embarazo la segunda causa $(71,6 \%)$, mientras que la oposición de la pareja al uso de algún método anticonceptivo determinó un $22,5 \%$ de necesidad insatisfecha en el uso de métodos anticonceptivos ${ }^{(18)}$.

Una mala información, mitos o creencias acerca de los métodos anticonceptivos son también una barrera para un uso adecuado. Son muy claros los mitos que existen acerca del dispositivo intrauterino, pues más de una mujer señala que es un método abortivo, que "falla mucho" y que produce infecciones. $Y$ es lamentable que en algunos casos estas barreras sean propiciadas por los mismos profesionales de salud quienes por desconocimiento, o por una determinada posición ideológica sustentan estas afirmaciones erradas. Es muy conocido que dentro de las razones religiosas hay una marcada oposición al uso de métodos anticonceptivos, a los cuales se les señala como abortivos, y se prioriza el uso de métodos llamados naturales, que en realidad son de abstinencia periódica y cuyo índice de falla es muy elevado.

En el Perú, la necesidad insatisfecha de PF habría disminuido de 10,2 \% (ENDES 2000) a 6,1\% (ENDES 2011). Sin embargo, debemos tener en cuenta que la mayor demanda insatisfecha se presenta en los quintiles de mayor pobreza $(9,9 \%)$ así como en las zonas de la selva $(9,7 \%)$ y en el área rural $(7,5 \%)^{(19)}$, justamente donde los problemas de salud reproductiva, como la morbilidad y mortalidad materna, son mayores.

\section{PLANIFICACIÓN FAMILIAR Y FECUNDIDAD}

En el último siglo el crecimiento poblacional ha sido bastante rápido, sobre todo en los países con menor desarrollo. Ello contrasta con el hecho que desde fines del siglo XIX y sobre todo empezando el siglo XX la fecundidad empezó a bajar drásticamente en países desarrollados, particualrmente en Europa, mientras que a fines de los años sesenta este fenómeno empezó a ocurrir en América Latina. Es decir, estaremos asistiendo a la disminución en el mundo de la población joven, y un incremento de la población adulta mayor.

Si bien el crecimiento demográfico tiene a la mortalidad y migración de la población como dos de sus componentes importantes, debemos reconocer que también está definido por los cambios en la fecundidad. J. Bongaarts, demostró que la diferencia en la fecundidad de las poblaciones se debe mayormente a la variación de cuatro variables principales: la nupcialidad, el uso de anticonceptivos, la infecundidad posparto y el aborto inducido ${ }^{(20)}$.

En el caso del Perú, en el período entre los años 1950 y 1965 las familias tenían un promedio de casi siete hijos. Treinta años después, entre los años 1990 y 1995, el promedio de la tasa global de fecundidad descendió a 3,3 ${ }^{(21)}$. Las encuestas demográficas y de salud recientes (ENDES 1986, 2000, 2012) señalan que la tasa global de fecundidad descendió significativamente de 4,1 (1986) a 2,9 hijos por mujer (2000) y a 2,6 en el año $2012^{(22)}$. 
La prevalencia actual del uso de métodos anticonceptivos ha variado entre la ENDES del año 2000 (68,9\%) y la ENDES del 2012 (75,5\%). Sin embargo, el uso de métodos modernos se ha mantenido sin modificación en estos 12 años, siendo un $51 \%$ del total ${ }^{(22)}$.

El descenso de la fecundidad aún no responde a las expectativas del número de hijos que las mujeres quieren tener, ya que la tasa global de fecundidad es de $2,6^{(22)}$; si todos los embarazos no deseados pudiesen ser prevenidos la tasa global de fecundidad sería mucho menor. Las mujeres de zonas urbanas, y las jóvenes con mayor nivel educativo son las que menor número de hijos quieren tener, pero en el sector rural la brecha entre el número de hijos que se tiene y el número de hijos deseados es más amplia.

\section{CONCLUSIONES}

Si bien la población mundial envejece, no podemos dejar de reconocer que hacer uso de la PF es una estrategia que las personas tienen a su alcance para gozar no solo de una adecuada salud sexual y reproductiva, sino también de una herramienta fundamental para su propio desarrollo y el de la sociedad. Derecho que debe ser respetado.

Por un tema de equidad en salud, no solo debemos disminuir la necesidad insatisfecha de PF, sino también reducir la brecha existente entre los métodos anticonceptivos modernos ofrecidos por el sector privado y el sector público. Deuda de muchos años que debe ser asumida totalmente por el Estado.

Es fundamental y deber del Estado tener políticas claras de PF, respetar la autodeterminación de hombres y mujeres cuando deciden planificar su vida reproductiva, $y$ el derecho que tienen de no tener interferencias en su decisión, así como en el acceso y disponibilidad a la más amplia gama de métodos anticonceptivos.

Fuente de financiamiento: autofinanciado.

Conflictos de interés: el autor declara no tener conflictos de interés en la publicación de este artículo.

\section{REFERENCIAS BIBLIOGRÁFICAS}

1. Herrero J, Valdés O, Cabero C. Anticoncepción: consideraciones generales. Clasificación de los métodos anticonceptivos. Técnicas, indicaciones y resultados de la anticoncepción y planificación familiar. En: Cabero RL, Director, Cabrillo RE, Coordinador General, Bajo AJ, et al. Tratado de Ginecología y Obstetricia Ginecología y Medicina de la Reproducción. 2a Ed. Madrid: Sociedad Española de Ginecología y Obstetricia; 2012: p 615-9.

2. Díaz Alonso G. Historia de la anticoncepción. Rev Cubana Med Gen Integr. 1995;11(2):192-4.

3. Naciones Unidas. Informe de la Conferencia Internacional sobre Población y Desarrollo. El Cairo Egipto, 5-13 Septiembre de 1994. Nueva York: Naciones Unidas; 1995.

4. Perú, Congreso Constituyente Democrático. Constitución Política del Perú. Título I: De la Persona y la Sociedad, Cap. II De los Derechos Sociales y Económicos Art. 6. Lima, Perú; 1993.

5. The Alan Guttmacher Institute (AGI). Aborto clandestino: una realidad latinoamericana. Nueva York: AGI; 1994.

6. Sing S, Wulf D. Niveles estimados de aborto en países latinoamericanos. International Family Planning Perspectives. Número Especial, 1994:3-13.

7. OMS. Atención para un aborto sin riesgos: Fundamentos lógicos desde el punto de vista de la salud pública y de los derechos humanos. En: Aborto sin riesgos: Guía Técnica y de Políticas para Sistemas de Salud. 2 ${ }^{\text {a }}$ ed. Ginebra: Organización Mundial de la Salud; 2012. p. 17-26.

8. Perú, Ministerio de Salud, Dirección General de Epidemiología. Principales causas de muerte materna en el Perú. En: La mortalidad materna en el Perú 2002 a 2011. Lima: MINSA; 2013. p. 55-60.

9. Espíndola E, León A. La deserción escolar en América Latina: un tema prioritario para la agenda regional. Revista Iberoamericana de Educación. 2002;(30):39-62.

10. Barot S. Back to Basics: The Rationale for increased Funds for International Family Planning. Guttmacher Policy
Review. 2008:11(3):16-7.

11. Speidel JJ, Sinding S, Gillespie D, Maguire E, Neuse M. Making the Case for U.S. International Family Planning Assistance. Baltimore, MD: The Bill and Melinda Gates Institute for Population and Reproductive Health; 2009.

12. Reproductive Health Supplies Coalition (RHSC). Contraceptive Projections and the Donor Gap. Meeting the Challenge. Belgium: RHSC; 2009.

13. United Nations Population Fund (UNFPA). El bono demográfico Regional en el Perú. En: Programa Conjunto Promoción del empleo y el emprendimiento de jóvenes y Gestión de la Migración Laboral Internacional Juvenil. Lima: UNFPA; 2012. p. 12-21.

14. Bloom DE, Williamson JG. Demographic Transitions and Economic Miracles in Emerging Asia. World Bank Economic Review. 1998;12(3):419-55.

15. Reducing unmet need for family planning: Evidence based strategies and approaches. Outlook. 2008;25(1):1-7. 
16. Singh S, Darroch JE. Adding it up: Costs and benefits of Contraceptive Services. Estimates for 2012. New York: Guttmacher Institute and United Nations Population Fund (UNFPA); 2012. p. 1-18.

17. Miranda JJ, Yamin AE. ¿Políticas de salud y salud politizada? Un análisis de las políticas de salud sexual y reproductiva en Perú desde la perspectiva de la ética médica, calidad de atención y derechos humanos. Cad. Saúde Pública. 2008;24(1):7-15.

18. $\mathrm{Ku}$ E. Causas de necesidades insatisfechas en planificación familiar en mujeres con embarazos no deseados del Instituto materno Perinatal. Rev Peru Epidemiol. 2010;14(2):124-32.

19. Instituto Nacional de Estadística e Informática (INEI). Perú: Encuesta Demográfica y de Salud Familiar 2011. Nacional y Departamental. Lima: INEI; 2012.

20. Bay G, Del Popolo F, Ferrando D. Determinantes Próximos de la fecundidad. Una aplicación a países latinoamericanos. CEPAL-Serie Población y Desarrollo; 2003;(43):725

21. Instituto Nacional de Estadística e Informática (INEI). Estimaciones y Proyecciones de Población 1950-2050.
Urbano Rural 1970-2025. Boletín de Análisis Demográfico. 2001;(35):1723.

22. Instituto Nacional de Estadística e Informática (INEI). Perú, Encuesta Demográfica y de Salud Familiar 2012. Nacional y Departamental. Lima: INEI; 2013.

Correspondencia: Miguel Gutiérrez

Dirección: Av. La Fontana 904 Urb. La Riviera de Monterrico. La Molina, Lima, Perú.

Teléfono: 999469168

Correo electrónico:mgutierrez@insap.org.pe

\section{Visite nuestra página en Facebook, www.facebook.com/rpmesp Infórmese sobre los eventos y los nuevos contenidos de la Revista Peruana de Medicina Experimental y Salud Pública}

\section{REVISTA PERUANA DE MEDICINA EXPERIMENTAL Y SALUD PÚBLICA \\ L} Difundiendo investigación en salud
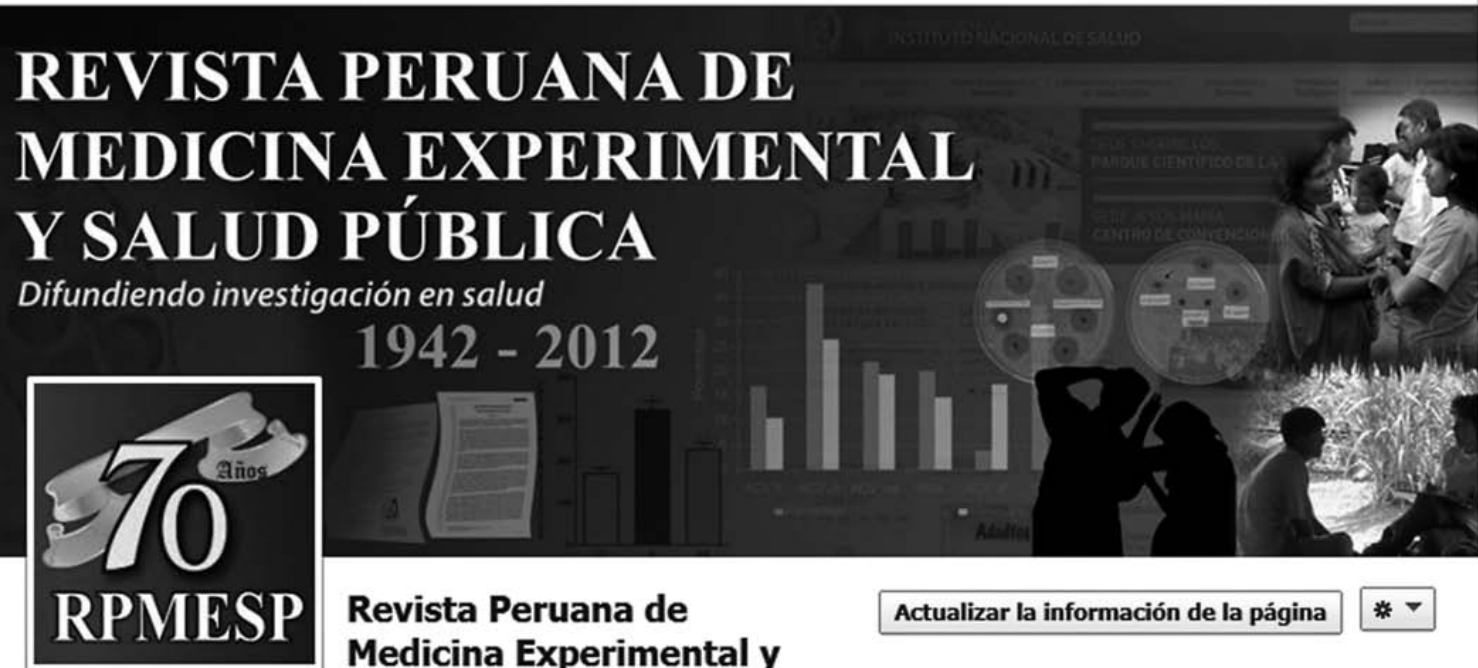

Revista Peruana de Medicina Experimental y

Salud Pública (OFICIAL)

A 842 personas les gusta esta página $\cdot 3$ personas están hablando sobre esto

Comunidad [?]

La Revista Peruana de Medicina Experimental y Salud

Publica es el órgano oficial de difusión científica del

Instituto Nacional de Salud

Información


Fotos

Me gusta

Notas 5

Eventos 